\section{Tecnologias da informação e comunicação em adolescentes, práticas parentais e percepção de clima escolar: Uma abordagem multinivel ${ }^{1}$}

\author{
Guilherme Welter Wendt \\ Marli Appel da Silva \\ Silvia Helena Koller
}

\section{RESUMO}

O presente estudo, quantitativo e transversal, avaliou o perfil de uso de tecnologias de informação e comunicação (TICs) entre adolescentes escolares, por meio de uma perspectiva multinível. Assim, possíveis diferenças no uso de TICs em relação à idade e ao sexo dos participantes bem como variações no perfil de uso de TICs quanto aos níveis de supervisão parental foram consideradas. Ainda, investigou-se em que medida o uso de TICs variou de acordo com as percepções sobre clima escolar por parte dos jovens. Participaram 367 adolescentes (55,6\% meninas) que responderam a um instrumento de autorrelato. Os resultados mostraram diferenças entre o gênero e a idade dos participantes quanto ao perfil de uso e no recebimento de práticas parentais sobre o uso de TICs. Diferenças quanto ao uso de TICs em relação com a percepção de clima escolar foram detectadas. Em síntese, o estudo sugere que a promoção de práticas educativas proativas de supervisão do uso de TICs pode trazer impactos positivos para o desenvolvimento sadio do adolescente.

Palavras-chave: Adolescência; tecnologia da informação; internet.

\begin{abstract}
Information and communication technologies in adolescents, parental practices and school climate perception: A multilevel approach

This cross-sectional study evaluated the information and communication technologies (ICTs) profile of high school adolescents through a multilevel perspective. Differences in ICTs usage according to age and sex, as well differences in the ICT profile in respect to parents' supervision were explored. Moreover, we investigated whether the use of ICTs changed according to adolescents' report on school climate. The participants were 367 adolescents (55.6\% girls) who responded to a self-reported measure. Age and gender differences were noted for ICTs profile, as well as for parental practices. Differences regarding ICTs profile and school climate were found. In summary, the study suggests that promoting proactive parental practices of supervision ICTs use might be associated to a healthier adolescent development.
\end{abstract}

Keywords: Adolescence; information technology; internet.

1 Artigo baseado na dissertação de mestrado de G W Wendt, apresentada à Universidade do Vale do Rio dos Sinos.

\section{Sobre os Autores}

G.W.W

http://orcid.org/0000-0002-90146120

Universidade Federal do Rio Grande do Sul

wendtguilherme@gmail.com

M.A.S

http://orcid.org/0000-0002-19630788

Pontifícia Universidade Católica do Rio Grande do Sul mappel@uol.com.br

\section{S.H.K}

http://orcid.org/0000-0001-91096674

Universidade Federal do Rio Grande do Sul

silvia.koller@gmail.com

\section{Direitos Autorais}

Este é um artigo de acesso aberto e pode ser reproduzido livremente, distribuído, transmitido ou modificado, por qualquer pessoa desde queu sado sem fins comerciais. 0 trabalho é disponibilizado sob a licença Creative Commons CC-BY-NC. 
0 objetivo do presente artigo foi analisar o perfil de uso de tecnologias de informação e comunicação (TICs) entre adolescentes escolares, incluindo a quantidade de horas utilizando a internet, o tipo de supervisão parental em relação às TICs, bem como os motivos e locais mais frequentes para acesso. Buscou-se comparar possíveis diferenças quanto à supervisão parental e uso de TICs entre meninos e meninas, além de explorar variações em relação aos adolescentes mais jovens (G1; 13 a 15 anos) e mais velhos (G2, 15 a 17 anos). Com efeito, diferenças entre adolescentes mais jovens e adolescentes mais velhos são particularmente úteis em estudos sobre fatores de risco e proteção, uma vez que ações preventivas podem ser planejadas de forma mais efetiva e específica (United Nations Children's Emergency Fund, 2016). Ainda, explorou-se em que medida o perfil de uso de TICs é importante na compreensão de distintas percepções de clima escolar por parte dos jovens. A análise de dados quanto ao uso de TICs em níveis múltiplos se assemelha à abordagem utilizada em pesquisas de larga escala sobre o papel da tecnologia na vida dos jovens, ou seja, não se limita apenas a aspectos individuais, mas leva em conta fatores múltiplos, conforme será explicado a seguir (Centro de Estudos sobre as Tecnologias da Informação - Cetic, 2018; Livingstone et al., 2015). Na dimensão individual, o perfil de uso de TICs poderia indicar possíveis fatores de risco e proteção para os adolescentes; no nível subsequente (social), incluem-se as inter-relações com as práticas de pais e educadores. Já o último nível de análise e de interpretação de dados (nível nacional) traz implicações mais amplas acerca de similaridades e diferenças no tocante às variáveis investigadas entre regiões e contextos distintos (Livingstone et al., 2015).

\subsection{0 uso das TICs por crianças e adolescentes}

Existem motivos importantes para a acurada compreensão tanto do modo como os jovens utilizam as TICs como em relação aos fatores parentais e escolares decorrentes do uso crescente da tecnologia na contemporaneidade. A partir dos dados provenientes de países desenvolvidos, constata-se que o uso de internet por parte de adolescentes é elevado (Fleming, 2018; Office of Communications, 2017). Os indivíduos inseridos na geração digital incorporam as TICs em praticamente todos os segmentos de suas vidas (relações interpessoais, performance acadêmica, organização de tarefas, dentre outras), razão pela qual passa a ser entendido que o desenvolvimento dos adolescentes ocorre permeado por tais tecnologias (Areepattamannil \& Khine, 2017; Könitzer et al., 2017; Wendt \& Lisboa, 2014).
Dados provenientes da Austrália indicam que, entre os anos de 2011 e 2012, houve um aumento na proporção de crianças acessando a internet (Australian Bureau of Statistics, 2012). Por exemplo, $79 \%$ dos participantes com idades entre 5 e 8 anos utilizaram a internet; já entre a faixa etária compreendida entre os 9 e 11 anos, aproximadamente $96 \%$ relataram utilizar a internet (Australian Bureau of Statistics, 2012). De modo interessante, não houve diferença no uso de internet entre participantes residindo em grandes centros vs. aqueles residentes em áreas remotas, bem como não houve diferença estatisticamente significativa entre aqueles que utilizam a internet na escola (2,2 milhões de usuários) vs. usuários residenciais de internet $(2,3$ milhões de usuários; Australian Bureau of Statistics, 2012). Outro exemplo vem de um estudo americano que constatou que $76 \%$ dos lares formados por adolescentes entre 12 e 17 anos possuíam conexão à internet de alta velocidade, cerca de $93 \%$ dos jovens haviam utilizado a internet regularmente e $75 \%$ possuíam telefone celular (Lenhart et al., 2010). Outra investigação americana, dessa vez incluindo participantes entre 8 e 18 anos, indicou que $66 \%$ dos jovens possuíam telefone celular próprio (Rideout et al., 2010). No Reino Unido, 99\% dos jovens entre 12 e 15 anos utilizaram a internet, em uma média de 21 horas por semana; $83 \%$ tinham o seu próprio smartfone; e $77 \%$ dos adolescentes dessa faixa etária jogavam online, em uma média de 12 horas por semana (Office of Communications, 2017).

No tocante aos modos de acesso e motivos para o uso de TICs, os jovens costumam conectar-se via computadores portáteis e tradicionais, telefones celulares e por meio de aparelhos de vídeo game. A conexão à internet possibilita o estabelecimento de conversações, auxilia na confecção de trabalhos escolares, favorece 0 acesso à música e à cultura televisiva, media o acesso à informação e compras, dentre outros (Lenhart et al., 2010). A naturalidade com a qual os jovens interagem com as TICs traz, segundo a opinião dos próprios usuários, benefícios para a ampliação da rede de contatos sociais (Zaremba et al., 2002).

No contexto brasileiro, também existem evidências robustas sobre elevado uso de TICs por parte de crianças e adolescentes. Os dados do Cetic (2018) indicaram que 93\% das crianças e adolescentes entre 9 e 17 anos, cujos pais possuíam nível secundário de educação, acessaram a internet no Brasil. Dentre os filhos de pais com nível fundamental de educação, a proporção foi de $76 \%$. Foram ainda detectadas disparidades em relação à faixa etária dos jovens, na qual indivíduos entre 15 e 17 anos acessaram mais a internet (93\%) quando comparados 
com adolescentes mais jovens (87\% no grupo com 13 e 14 anos, e $82 \%$ dentre os jovens com 11 e 12 anos; Cetic, 2018). Cerca de 2,2 milhões de jovens brasileiros entre 9 e 17 anos nunca utilizou a internet, o que corresponde a $8 \%$ do total de sujeitos nessa faixa etária. As principais explicações para o não acesso à internet incluíam falta de acesso no domicílio (9\%), sobretudo dentre jovens residentes em contextos rurais e da região Norte (Cetic, 2018).

Evidências mostram uma ascensão no uso de redes sociais por parte dos jovens. Dentre os adolescentes americanos que possuem acesso à internet, 73\% têm contas em redes sociais (Lenhart et al., 2010). No Reino Unido, cerca de três quartos dos participantes com idades entre 12 e 15 anos e com acesso à internet possuem contas em redes sociais (Office of Communications, 2017). Uma investigação com jovens brasileiros em situação de vulnerabilidade social, incluindo 60 participantes com idades entre 14 e 18 anos, mostrou que $94,7 \%$ tinham conta ativa no Facebook (Oliveira et al., 2017). O estudo indicou ainda que os participantes costumam aprender a utilizar a internet sem auxílio de adultos, sendo que os principais motivos para o uso são a comunicação com pares e entretenimento (e.g., jogos; Oliveira et al., 2017). Apenas $5 \%$ dos jovens afirmaram não ter utilizado a internet nos últimos 12 meses anteriores à pesquisa, relatando motivos relacionados ao alto custo e, ainda, por considerarem arriscado o uso.

\subsection{Além da dimensão individual: TICs, mediação paren- tal e impactos na escola}

Embora a literatura discutida até então tenha dado ênfase em variáveis sociodemográficas associadas com o uso de TICs, sabe-se que, durante a adolescência, uma parte significativa do tempo dos jovens é vivenciada na escola (Zaremba et al., 2010). Com efeito, a incorporação das TICs tem tido repercussões específicas no campo do ensino, desafiando a comunidade escolar na adoção de novas práticas (Oliveira, 2017). Pesquisadores buscam analisar a associação entre a percepção de clima escolar com variáveis relacionadas com as TICs, incluindo uso abusivo de internet (Jia et al., 2009; Li et al. , 2016). Conforme Li et al. (2016), o clima escolar - conceito que abarca as propriedades do ambiente escolar que afetam o comportamento dos estudantes - exerce um papel crucial no desenvolvimento ou não de problemas comportamentais.

Dentre os principais problemas de comportamento resultantes de uma percepção de clima escolar negativa, inclui-se adição à internet, conceito que alude ao uso excessivo e prejudicial de internet (Levesque, 2018; Li et al., 2016). Contando com uma amostra de 2758 adolescentes chineses $(M=13,53, D P=1,06)$, um estudo indicou que, após controlar as variáveis sociodemográficas, o clima escolar negativo associou-se com a adição à internet e que a relação foi mediada pela associação com colegas problemáticos (Li et al., 2016). Em escolas com clima escolar negativo, há maior probabilidade de que os jovens se sintam isolados ou rejeitados, o que facilitaria a afiliação com pares problemáticos na busca por aceitação (Rudasill et al., 2014; Yen et al., 2009).

É comum que os pais utilizem estratégias e controle para mediar as atividades online de seus filhos, incluindo restrição de uso, supervisão do uso e discussões acerca dos riscos presentes no mundo digital. Duas estratégias gerais de mediação parental podem ser identificadas em relação ao uso de TICs por parte de crianças e adolescentes: mediação restritiva e mediação instrutiva. A mediação restritiva refere-se à regulação do uso de mídia por meio da criação de regras. A mediação instrutiva refere-se aos esforços ativos dos pais para interpretar e traduzir as informações obtidas pelos filhos através das TICs (Livingstone \& Helsper, 2008). Existe uma tendência maior para supervisão de comportamentos e atitudes online por parte de famílias com maior poder aquisitivo; bem como meninas, no geral, são mais supervisionadas quanto às suas atividades online do que meninos (Livingstone \& Helsper, 2008). Ainda, existe um padrão adotado pelos pais e/ou responsáveis no tocante à supervisão das atividades online dos filhos que segue uma curva em $U$, ou seja, a mediação parental aumenta até o início da adolescência, comumente decrescendo após esse período (Hasebrink et al., 2009). Todavia, não está claro se determinado tipo de estratégia de monitoramento parental diante do uso de TICs é particularmente eficaz para reduzir os riscos da exposição dos jovens às TICs, bem como pouco se sabe que fatores podem promover resiliência para lidar com situações adversas no contexto digital (Kirwil, 2009).

\subsection{0 presente estudo}

Conforme destacado anteriormente, este estudo buscou analisar o perfil de uso de TICs entre adolescentes escolares, comparando os dados entre meninos e meninas e entre adolescentes mais jovens e adolescentes mais velhos. Um segundo objetivo do estudo foi de investigar possíveis diferenças entre uso de TICs de acordo com 
o nível de supervisão parental e com a percepção de clima escolar por parte dos alunos. Hipotetizou-se que adolescentes mais velhos teriam um maior uso e uma menor supervisão parental em relação ao uso de TICs (Australian Bureau of Statistics, 2012; Hasebrink et al., 2009). Ainda, o presente estudo anteviu que meninas receberiam maior supervisão parental no tocante ao uso de TICs em comparação com meninos (Hasebrink et al., 2009). Não foram estabelecidas hipóteses a priori em relação à percepção de clima escolar e o perfil de TICs por parte dos adolescentes.

\section{MÉTODO}

\subsection{DELINEAMENTO E PARTICIPANTES}

Trata-se de um delineamento quantitativo, transversal, de levantamento e correlacional. Participaram do estudo 367 adolescentes (55,6\% meninas), com idades variando entre 13 até 17 anos $(M=14,76, D P=1,40)$, estudantes de escolas públicas e privadas localizadas na região metropolitana de Porto Alegre. A amostra foi composta por dois grupos, sendo um formado por adolescentes com até 15 anos (G1) e o outro por adolescentes com idade superior a 15 anos (G2). No G1, que compreendeu participantes com idades entre 13 e 15 anos, a média de idade foi 13,58 anos (DP $=0,64$ ) e $54,3 \%$ eram meninas. 0 $\mathrm{G} 2$, envolvendo aqueles com mais de 15 e menos de 18 anos, apresentou uma média de idade de 15,96 anos (DP $=0,82$ ) e foi formado por $56,9 \%$ de meninas.

\subsection{INSTRUMENTOS}

0 instrumento utilizado na presente investigação foi elaborado pelos autores, e continha questões como idade do participante, escola e série, bem como sobre comportamentos em relação às TICs (tempo médio utilizando internet, finalidade e local do uso, entre outros). Dados demográficos como a escolaridade e profissão dos pais e ou responsáveis, assim como aspectos relativos à moradia (com quem reside, se possui irmãos), também foram coletados. Juntamente do questionário biossociodemográfico, foram incluídas oito questões sobre a percepção dos alunos sobre o clima escolar $(a=0,66)$, apresentadas em uma escala variando de 1 (sim, sempre) até 3 pontos (não); escores mais elevados indicam percepção pior do ambiente escolar (escore máximo possível = 24; Genta et al., 2009). Ainda, a percepção dos adolescentes em relação as atitudes dos pais frente as TICs foram analisadas em três questões, apresentadas em uma escala variando de 1 (sim, sempre) até 3 pontos (nunca); novamente, escores mais elevados indicam menor monitoramento parental em relação ao uso de TICs ( $a=0,64$; Genta et al., 2009). Detalhes acerca dos procedimentos de adaptação e tradução dos instrumentos encontram-se disponíveis em publicações prévias (e.g., Wendt, 2012).

\subsection{PROCEDIMENTOS}

O tamanho da amostra foi calculado utilizando o programa $G *$ Power, que indicou haver a necessidade de, no mínimo, 148 participantes por grupo de modo a atingir um poder de $99 \%$ e um nível de significância igual ou menor a 0,05 (Faul et al., 2007). Como critério de inclusão, todos os participantes deveriam estar regularmente matriculados e ter utilizado a internet ao menos uma vez nos últimos seis meses. De acordo com as diretrizes envolvendo a pesquisa com seres humanos, esse estudo seguiu todos os cuidados éticos necessários, garantindo ainda o anonimato no tratamento dos dados e na divulgação dos achados dessa pesquisa. Do mesmo modo, a investigação atentou para o princípio de bem-estar e da premissa em não causar dano aos participantes. Além disso, o estudo foi aprovado pelo Comitê de Ética em Pesquisa da Universidade do Vale do Rio dos Sinos (Parecer n. 11184). Inicialmente, sete escolas foram contatadas para apresentação do projeto e obtenção de aprovação para a coleta de dados. Obtevese a autorização de quatro instituições escolares, que assinaram a carta de anuência. Posteriormente, foi entregue o Termo de Consentimento Livre e Esclarecido, instrumento que solicitou a assinatura dos pais/ responsáveis e também do próprio estudante participante do estudo. Os instrumentos foram respondidos individualmente, em sala de aula e em sessão única, com a presença do professor responsável pela disciplina e com duração média de 30 minutos. Caso necessário, foram fornecidas instruções específicas para os participantes, de modo individual. Todos os participantes foram informados sobre a possibilidade de recusa em participar da investigação; e, no caso da ocorrência de qualquer desconforto ou sofrimento emocional provocado em decorrência do estudo, o pesquisador e os auxiliares de pesquisa foram instruídos a realizar os devidos encaminhamentos.

\subsection{ANÁLISE DOS DADOS}


Os dados foram tabulados e analisados no Statistical Package for the Social Sciences - SSPS versão 22.0. Análises descritivas, de caráter exploratório, foram conduzidas para a checagem de possíveis erros cometidos durante a fase de tabulação e também foram analisados os outliers (casos extremos). 0 teste de quiquadrado $(X 2)$ foi utilizado para verificar diferenças entre variáveis categóricas, sendo apresentadas as razões de chance (RC) e os respectivos intervalos de confiança (IC) na ordem de 95\%; e, nas variáveis contínuas, utilizou-se o teste $t$ de Student. As diferenças investigadas foram consideradas significativas se $p$ apresentasse valor igual ou inferior a 0,05 .

\section{RESULTADOS}

A maioria dos participantes deste estudo residia com os pais $(90,7 \%)$ e $6,8 \%$ afirmaram residir com parentes. Além disso, $86,3 \%$ declararam ter irmãos, variando entre 1 a 6 irmãos $(M=2,08, D P=2,42)$. A seguir, serão apresentados os resultados do estudo de acordo com os objetivos propostos.

\subsection{Nivel individual de análise: o perfil do uso de TICS's}

Em relação ao uso de TICs, os que possuíam computadores em casa representaram mais de três quartos da amostra (84,5\%), sendo que $56,5 \%$ dispunham de um equipamento de uso exclusivo. Em relação ao telefone celular, $95,1 \%$ afirmaram a posse de aparelho próprio. Sobre a habilidade em utilizar computadores, mais da metade da amostra $(59,7 \%)$ relatou satisfação com suas habilidades e $29 \%$ consideraram como excelente suas capacidades para o manejo da ferramenta. Apenas $11 \%$ perceberam suas habilidades como não muito boas para uso desse equipamento.

No que diz respeito ao acesso à internet, $67,2 \%$ relataram uso diário, $14,2 \%$ afirmaram utilizar de três a cinco vezes por semana, seguido de $11,2 \%$ que acessam a rede até duas vezes por semana. 0 tempo gasto, em média, utilizando a internet foi de 2,96 horas por dia $(D P=3,10)$, variando de 0 a 20 horas diárias, posto que não existiram diferenças quanto ao uso da internet em relação ao sexo do participante; mas, sim, em relação à faixa etária $(t(349)=-3,03, p<0,05)$, uma vez que adolescentes mais velhos (G2) gastaram, diariamente, um tempo significativamente superior na internet quando comparados aos mais novos (G1).
Com relação aos locais para uso da internet, observouse que a maioria dos participantes $(61,8 \%)$ não acessou a internet a partir da escola. Os locais mais comuns para o acesso à rede reportados foram: do quarto $(62,5 \%)$; em casa, fora do quarto $(50,4 \%)$; via aparelho celular $(44,7 \%)$; casa de um amigo (42,7\%); e em uma lan house $(27,7 \%)$. Não foram encontradas diferenças significativas entre os sexos em relação ao acesso à internet, com exceção do uso no celular, que foi mais comum entre as meninas $(X 2(2)=6,80, p<0,05 ; \mathrm{RC}=1,26 ; 95 \% \mathrm{IC}=1,05-1,51)$. Os adolescentes mais velhos (G2) diferiram do $\mathrm{G} 1 \mathrm{em}$ relação ao uso da internet no quarto $(X 2(1)=6,84, p<$ $0,05 ; \mathrm{RC}=1,35 ; 95 \% \mathrm{IC}=1,06-1,71)$, na escola $(X 2(1)=$ $41,05, p<0,001 ; R C=1,96 ; 95 \%$ IC $=1,63-2,36$ ), na casa de um amigo $(X 2(1)=10,24, p<0,05 ; \mathrm{RC}=1,37 ; 95 \%$ IC $=1,11-1,68)$, no trabalho $(X 2(1)=14,59, p<0,001 ; \mathrm{RC}=$ $1,73 ; 95 \% \mathrm{IC}=1,41-2,12)$, na casa de parentes $(X 2(1)=$ $4,13, p<0,05 ; \mathrm{RC}=1,24 ; 95 \% \mathrm{IC}=1,01-1,52)$, bem como o uso em casa, fora do quarto $(X 2(1)=5,60, p<0,05$; RC $=1,29 ; 95 \%$ IC $=1,04-1,59)$. Em todas essas ocasiões, os adolescentes mais velhos apresentaram frequência superior de uso da internet. O G1, todavia, apresentou maior uso da internet em lan houses em relação ao G2 $(X 2(2)=7,93, p<0,05 ; \mathrm{RC}=0,70 ; 95 \% \mathrm{IC}=0,53-0,93)$.

Quando questionados sobre os motivos para o uso da internet, os resultados apontaram que $73,3 \%$ dos participantes utilizaram a internet para a realização de trabalhos escolares, $69,5 \%$ para utilização de programas de conversas instantâneas, $66,1 \%$ para a realização de downloads, $65 \%$ com o propósito de visitar sites, $45,5 \%$ para enviar e receber e-mails, $44,6 \%$ para jogos online e $17,6 \%$ para compras. Meninos e meninas diferiram em seus motivos para uso da internet. As meninas declararam o uso para a realização de trabalhos escolares em frequência superior a declarada pelos meninos $(X 2(1)=$ $18,32, p<0,001 ; R C=1,69 ; 95 \% I C=1,27-2,21$ ), enquanto os meninos estiveram mais envolvidos em jogos online $(X 2(1)=13,29, p<0,001 ; \mathrm{RC}=1,53 ; 95 \% \mathrm{IC}=1,21-1,93)$.

Diferenças significativas foram constatadas também entre os adolescentes mais jovens e os mais velhos. Participantes do $\mathrm{G} 2$ utilizaram mais a internet para visitar sites $(X 2(2)=28,98, p<0,001 ; \mathrm{RC}=1,97 ; 95 \% \mathrm{IC}=1,48$ $2,62)$, enviar e receber e-mails $(X 2(2)=26,41, p<0,001$; $\mathrm{RC}=1,70 ; 95 \% \mathrm{IC}=1,37-2,10)$, para o uso de softwares de conversas instantâneas $(X 2(1)=4,92, p<0,05 ; R C=1,31$; $95 \%$ IC $=1,01-1,69)$, trabalhos escolares $(X 2(1)=7,88, p$ $<0,05 ; \mathrm{RC}=1,49 ; 95 \% \mathrm{IC}=1,09-1,92)$, download de filmes, programas e músicas $(X 2(1)=12,05, p<0,001 ; R C=1,52$; $95 \%$ IC $=1,17-1,97)$, bem como compras online $(X 2(1)=$ $5,41, p<0,05 ; R C=1,34 ; 95 \% I C=1,07-1,68)$. Além disso, 
86,6\% dos participantes afirmaram que já tiveram ou ainda têm uma conta em sites de rede social. Os meninos apresentaram um percentual levemente superior em relação a essa variável $(87,1 \%)$ quando comparados com as meninas $(86,3 \%)$.

\subsection{Nivel social de análise: o monitoramento dos pais e/ ou responsáveis sobre o uso de TICs}

Sobre percepção dos filhos em relação às atitudes dos pais no estabelecimento de regras e monitoramento das páginas/conteúdo que os adolescentes visualizam na internet, não foram encontradas diferenças entre meninos e meninas. Porém, no que tange ao fornecimento de conselhos sobre o uso das TICs, encontrou-se diferenças significativas entre os sexos $(X 2(2)=21,13, p<0,001$; $\mathrm{RC}=1,66 ; 95 \% \mathrm{IC}=1,25-2,20)$, no qual as meninas informaram ter recebido mais conselhos de seus pais que os meninos. As três atitudes dos pais - estabelecimento de regras, monitoramento de páginas visualizadas e fornecimento de conselhos - foram significativamente mais frequentes para os adolescentes mais jovens (G1). Os valores do teste de qui-quadrado, graus de liberdade e níveis de significância foram, respectivamente: $X 2(2)=$ $26,69, p<0,001, X 2(2)=16,00, p<0,001$ e $X 2(2)=22,43$, $p<0,001$. Não foi possível o cálculo de RC uma vez que as questões sobre estabelecimento de regras possuíam três opções de resposta (e.g., às vezes, sempre e nunca). Quando as atitudes dos pais foram combinadas para gerar um escore único, constatou-se que, quanto menor a frequência do estabelecimento de regras, monitoração de páginas e fornecimento de conselhos, maior foi o uso semanal de internet por parte dos adolescentes $(r=0,13$, $p<0,05)$.

\subsection{Nivel social de análise: o uso de TICs e a escola}

Inicialmente, constatou-se diferenças entre meninos e meninas em relação à percepção escolar, mas não entre as faixas etárias (e.g., G1 vs. G2). Meninos apresentaram melhor percepção da escola $(M=14,08, D P=3,00)$ que meninas $(M=14,75, D P=2,84 ; t(358)=-2,17, p<0,05)$. Diferenças nos níveis de percepção do clima escolar em virtude das atividades realizadas internet foram constatadas, sobretudo as correspondentes a navegar sites, entrar em salas de bate-papo, realizar trabalhos de escola e utilizar redes sociais. Assim, quem não utilizou a internet para navegar em sites teve uma pior percepção da escola $(M=15,02, D P=2,96)$ quando comparados aos jovens que utilizaram a internet para tal fim $(M=14,17$,
$D P=2,88 ; t(356)=-2,61, p<0,05)$. Já os jovens que não utilizaram a internet para entrar em salas de bate-papo tiveram uma melhor percepção da escola $(M=14,31, D P$ $=2,91$ ) quando comparados aos jovens que utilizaram a internet para entrar em salas de bate-papo $(M=15,42$, $D P=2,88 ; t(356)=2,42, p<0,05)$. Ademais, quem não utilizou a internet para trabalhos da escola teve uma pior percepção de clima escolar $(M=15,26, D P=2,99)$ quando comparados aos jovens que utilizaram a internet para tal fim $(M=14,17, D P=2,87 ; t(356)=-3,13, p<0,05)$. Ainda, quem não utilizou a internet com o propósito de acessar redes sociais teve uma pior percepção de clima escolar $(M=14,68, D P=2,87)$ quando comparados aos jovens que utilizaram a internet para tal fim $(M=14,02, D P=3,03$; $t(356)=-2,00, p<0,05)$.

Em relação ao local de acesso à internet, houve diferença apenas no tocante ao acesso residencial, fora do dormitório. Nesse aspecto, adolescentes que utilizaram a internet fora do quarto tiveram uma melhor percepção escolar $(M=13,94, D P=2,88)$ do que aqueles que acessaram a internet dentro do quarto $(M=15,00$, $D P=2,91 ; t(356)=-3,43, p<0,001)$. Quando as respostas sobre percepção da escola foram combinadas para gerar um escore único, constatou-se que não houve associação significativa entre uma melhor ou pior percepção de clima escolar com uso de internet por parte dos adolescentes $(p>0,05)$.

\section{DISCUSSÃO}

Um dos objetivos do presente estudo foi investigar o perfil de uso de TICs entre adolescentes escolares, comparando possíveis diferenças entre meninos e meninas e entre adolescentes mais jovens vs. adolescentes mais velhos. A análise dos resultados sociodemográficos mostrou que o uso de TICs se faz presente no cotidiano da amostra, seja através do uso da internet, seja pela posse de telefones celulares e computadores. A média diária utilizando a internet encontrada na investigação coincide com o tempo médio reportado por adolescentes Britânicos (e.g., 3 horas diárias; Office of Communications, 2017).

O presente estudo hipotetizou que participantes mais velhos (G2) teriam um maior uso de TICs. Adolescentes pertencentes ao G2 diferiram significativamente dos pertencentes ao G1 na frequência do uso como em relação ao perfil do uso, ocorrendo maior uso da internet em casa por parte do G2. Participantes mais velhos utilizaram mais a internet para visitar sites, enviar e receber 


\section{HET INTERACÃOEM
PSICOLOGIA}

Guilherme Welter Wendt, Marli Appel da Silva, Silvia Helena Koller

e-mails, utilizar programas de conversas instantâneas, realizar trabalhos escolares, efetuar compras e realizar downloads quando comparados ao G1. Em relação ao sexo, meninas utilizaram a internet para trabalhos escolares em frequência superior a declarada pelos meninos que, por sua vez, utilizaram mais a internet para jogos online. Esses dados são similares aos encontrados por Oliveira et al. (2017), cujos principais motivos para o uso de TICs foram: entrar em redes sociais, realizar downloads, estudar e enviar e-mails. Porém, Oliveira et al. (2017) não reportaram diferenças significativas quanto ao gênero. Ainda, as diferenças no perfil do uso de TICs entre adolescentes mais jovens vs. mais velhos parecem refletir tendências do cotidiano nessa faixa de idade em âmbitos nacionais e internacionais (Australian Bureau of Statistics, 2012; Cetic, 2018).

A presente investigação também propôs a hipótese de que o G2 e meninos apresentariam uma menor supervisão parental em relação ao uso de TICs. Tais hipóteses foram parcialmente suportadas. Assim, acerca das atitudes dos pais no que se refere ao estabelecimento de regras e monitoramento das páginas/conteúdo que os adolescentes visualizam na internet, não foram encontradas diferenças entre meninos e meninas. Porém, no que tange ao fornecimento de conselhos sobre o uso das TICs, encontrou-se diferenças significativas entre os sexos, sendo que as meninas informaram ter recebido mais conselhos de seus pais que os meninos. Novamente, tais resultados parecem refletir uma tendência observada em investigações anteriores (Australian Bureau of Statistics, 2012; Hasebrink et al., 2009).

Um último objetivo do estudo incluiu a investigação de possíveis associações entre o perfil e frequência de uso de TICs com a percepção de clima escolar. Não houve associação significativa entre uma melhor ou pior percepção escolar com a frequência de uso da internet por parte dos adolescentes, nem em relação ao tipo de supervisão parental e uma melhor ou pior percepção escolar, o que contraria alguns estudos (Jia et al., 2009; Li et al., 2016). O estudo de Li et al. (2016) teve como desfecho a variável adição à internet, componente não avaliado na presente investigação. Todavia, variações acerca da percepção do clima escolar ocorreram em virtude do perfil do uso de TICs. Assim, jovens que não utilizaram a internet para a visitação de páginas, acesso às redes sociais e para a realização de trabalhos escolares tiveram uma pior percepção do clima escolar quando comparados aos jovens que utilizaram a internet para tais finalidades. Ademais, aqueles que não utilizaram a internet para entrar em salas de bate-papo tiveram uma melhor percepção da escola quando comparados aos jovens que utilizaram a internet para entrar em salas de bate-papo. Finalmente, adolescentes que utilizaram a internet fora do quarto tiveram uma melhor percepção escolar do que aqueles que acessaram a internet dentro. Embora qualquer tipo de interpretação desses achados em relação à percepção de clima escolar seja meramente hipotético, um entendimento possível é que o maior uso da internet para visitar páginas possa fortalecer uma melhor percepção de clima escolar justamente por fortalecer uma rede de apoio social (Ellison et al., 2011; Pascarella \& Terenzini, 2005). Ainda, o uso da internet em locais públicos, fora do quarto do adolescente, pode indicar uma maior supervisão parental quanto ao uso de TICs ou mesmo um uso menos intenso, o que traria benefícios no domínio educacional (Li et al., 2016). Todavia, investigações futuras ainda são necessárias para melhor compreender a relação entre a frequência e o perfil de uso de TICs por parte de adolescentes brasileiros e suas implicações acadêmicas.

Portanto, os achados deste estudo sugeriram que os adolescentes mais velhos tenderam a receber menor frequência de supervisão parental que os mais jovens, bem como as meninas informaram ter recebido mais conselhos de seus pais que os meninos. Além disso, determinados tipos de uso da internet parecem influenciar a uma percepção mais positiva do clima escolar. A percepção do clima escolar pode ter um papel importante no desempenho acadêmico dos jovens (Ellison et al., 2011). Diante desse contexto, seria de valia a promoção de práticas educativas proativas voltadas aos pais em relação às suas práticas de supervisão parental para o uso da internet e TICs por parte dos jovens. Dessa forma, pode ser possível que a internet possa apresentar um uso mais presente na educação formal dos jovens. Vale ressaltar a importância da supervisão parental em todas as fases da adolescência, independente da idade e sexo dos jovens.

\subsection{Implicações e limitações do estudo}

Em uma perspectiva multinível, os dados aqui apresentados apresentam relativa similaridade com os reportados em nível nacional. Conforme o último relatório Cetic (2018), que incluiu mais de 23 mil domicílios das cinco regiões do Brasil, apontou que $90 \%$ dos jovens urbanos utilizaram a internet, contra $63 \%$ daqueles de áreas rurais. Porém, quando o quesito renda foi avaliado, $98 \%$ dos indivíduos de 9 e 17 anos das classes A e B utilizaram a internet, contra $70 \%$ das classes D e E. Considerando- 


\section{WL INTERACÃO EM
PSICOLOGIA}

se as particularidades da região Sul do Brasil, é possível ainda estabelecer paralelos entre o perfil de uso de TICs apresentado pelos participantes deste estudo com aqueles detectados em países desenvolvidos, como o Reino Unido (Office of Communications, 2017). Porém, os dados apontados pelo Cetic (2018) ainda indicam que a disparidade média de acesso à internet entre jovens brasileiros residentes em áreas urbanas vs. áreas rurais contraria indicadores de países desenvolvidos, como a Austrália (Australian Bureau of Statistics, 2012).

Segundo o Cetic (2018), é preciso garantir às crianças brasileiras o acesso à internet, uma vez que os adolescentes mais velhos (e.g., 15, 16 e 17 anos) acessam mais a internet (93\%) quando comparados aos mais jovens (e.g., 13 e 14 anos; 87\%). Uma das justificativas para a universalização do acesso à internet é a de que os jovens poderiam melhor desenvolver suas potencialidades de maneiras que não eram possíveis anteriormente (Ellison et al., 2011; Hasebrink et al., 2009). Ainda, a população jovem parece se beneficiar de modo especial da internet para exercer o protagonismo civil, engajamento cívico, aprendizado de línguas e conhecimento de outras culturas (Allen et al., 2018). Por outro lado, as TICs podem exacerbar alguns problemas, incluindo os fenômenos do cyberbullying, adição às TICs, exposição à publicidade imprópria e dificuldades de aprendizagem (Desmond, 2018; Li et al., 2016; Silva \& Silva, 2017).

É importante que pesquisadores, formuladores de políticas e os próprios pais aprendam quais estratégias são eficazes para o uso sadio das TICs (Kirwil, 2009). Sabe-se que quanto maior o envolvimento dos pais quanto ao uso das TICs, menor é a ocorrência de problemas decorrentes do mau uso da tecnologia (Livingstone \& Helsper, 2008). Todavia, cabe finalmente destacar que este estudo, embora apresente dados importantes, possui uma série de limitações. Ressalta-se que o delineamento transversal permite o cálculo apenas de associações entre variáveis, ficando a análise de causalidade comprometida. Futuros estudos sobre o uso de TICs por jovens, incluindo investigações longitudinais, poderão elucidar aspectos ainda pouco explorados no contexto nacional.

\section{DECLARAÇÃO DA CONTRIBUIÇÃO DOS AUTORES}

G. W. W. participou do delineamento do estudo, coleta e análise de dados, bem como estruturação do manuscrito. M. A. S. e S. H. K. participaram através da formulação ou desenvolvimento da proposta geral e objetivos do estudo, bem como colaboraram na redação e edições das versões do manuscrito.

\section{DECLARAÇÃO DE CONFLITOS DE INTERESSE}

Os autores declaram que não há conflitos de interesse no manuscrito submetido.

\section{REFERÊNCIAS}

Allen, L. R., Bogard, K., \& Yanish, T. (2018). Civic and citizenship attitudes. In R. Levesque (Ed.), Encyclopedia of Adolescence (2nd ed.) (pp. 600-606). Springer International Publishing. https:/doi.org/10.1007/9783-319-33228-4

Areepattamannil, S., \& Khine, M. S. (2017). Early adolescents' use of information and communication technologies (ICTs) for social communication in 20 countries: Examining the roles of ICT-related behavioral and motivational characteristics. Computers in Human Behavior, 73, 263-272. https:/doi.org/10.1016/j. chb.2017.03.058

Australian Bureau of Statistics. (2012). 4901.0 Children's participation in cultural and leisure activities, Australia. http://www.abs.gov.au/ausstats/abs@.nsf/ Products/4901.0 Apr_2012 Main+Features

Internet+and+mobile+phone/OpenDocument

Centro de Estudos sobre as Tecnologias da Informação - Cetic. (2018). Pesquisa sobre o uso da Internet por crianças e adolescentes no Brasil. TIC Kids Online Brasil 2017. https://www.cetic.br/media/ docs/publicacoes/2/tic_kids_online_2017_livro_ eletronico.pdf

Desmond, D. (2018). Advertising: Do not buy that. In R. Levesque (Ed.), Encyclopedia of Adolescence (2nd ed.) (pp. 123-129). Springer International Publishing. https:/doi.org/10.1007/978-3-319-33228-4 
Ellison, N. B., Steinfield, C., \& Lampe, C. (2011). Connection strategies: Social capital implications of Facebook-enabled communication practices. New Media \& Society, 13(6), 873-892. https:/doi. org/10.1177/1461444810385389

Faul, F., Erdfelder, E., Lang, A. G., \& Buchner, A. (2007). G*Power 3: A flexible statistical power analysis program for the social, behavioral, and biomedical sciences. Behavior Research Methods, 39(2), 175191. https:/doi.org/10.3758/bf03193146

Fleming, M. J. (2018). Cyberspace safety. In R. Levesque (Ed.), Encyclopedia of Adolescence (2nd ed,) (pp. 843852). Springer International Publishing. https:/doi. org/10.1007/978-3-319-33228-4

Genta, M. L., Brighi, A., \& Guarini, A. (2009). European project on bullying and cyberbullying granted by Daphne II programme. Zeitschrift für Psychologie/ Journal of Psychology, 217(4), 233. https:/doi. org/10.1027/0044-3409.217.4.233

Hasebrink, U., Livingstone, S., Haddon, L., \& Ólafsson, K. (2009). Comparing children's online opportunities and risks across Europe: Cross-national comparisons for EU Kids Online. EU Kids Online.

Jia, Y., Way, N., Ling, G., Yoshikawa, H., Chen, X., Hughes, D., Ke, X., Lu, Z. (2009). The influence of student perceptions of school climate on socioemotional and academic adjustment: A comparison of Chinese and American adolescents. Child Development, 80(5), 1514-1530. https:/doi.org/10.1111/j.14678624.2009.01348.x

Kirwil, L. (2009). Parental mediation of children's internet use in different European countries. Journal of Children and Media, 3(4), 394-409. https:/doi. org/10.1080/17482790903233440

Könitzer, B., Jeker, F., \& Waller, G. (2017). Young Children (0-8) and digital technology: A qualitative exploratory study - National report Switzerland based on the 2016 survey. Zurich: Zurich University of Applied Sciences.
Lenhart, A., Purcell, K., Smith, A., \& Zickuhr, K. (2010). Social media and young adults: Social media and mobile internet use among teens and young adults. Washington, DC: Pew Research Center.

Levesque, R. (2018). Addiction. In R. Levesque (Ed.), Encyclopedia of Adolescence (2nd Edition) (pp. 7982). Cham: Springer International Publishing. https:/ doi.org/10.1007/978-3-319-33228-4

Li, D., Zhou, Y., Li, X., \& Zhou, Z. (2016). Perceived school climate and adolescent Internet addiction: The mediating role of deviant peer affiliation and the moderating role of effortful control. Computers in Human Behavior, 60, 54-61. https:/doi.org/10.1016/j. chb.2016.02.015

Livingstone, S., \& Helsper, E. J. (2008). Parental mediation of children's internet use. Journal of Broadcasting \& Electronic Media, 52(4), 581-599. https:/doi. org/10.1080/08838150802437396

Livingstone, S., Mascheroni, G., \& Staksrud, E. (2015). Developing a framework for researching children's online risks and opportunities in Europe. EU Kids Online. http://eprints.Ise.ac.uk/id/eprint/64470

Office of Communications. (2017). Children and parents: Media use and attitudes report. Ofcom. https://www. ofcom.org.uk/research-and-data/media-literacyresearch/childrens/children-parents-2017

Oliveira, E. S. G. (2017). Adolescência, internet e tempo: Desafios para a Educação. Educar em Revista, 33(64), 283-298. https:/doi.org/10.1590/0104-4060.47048

Oliveira, M. P. M. T., Cintra, L. A. D., Bedoian, G., Nascimento, R., Ferré, R. R., \& Silva, M. T. A. (2017). Uso de internet e de jogos eletrônicos entre adolescentes em situação de vulnerabilidade social. Temas em Psicologia, 25(3), 1167-1183. https:/doi.org/10.9788/TP2017.3-13Pt

Pascarella, E. T., \& Terenzini, P. T. (2005). How college affects students. Jossey-Bass. https:/doi. org/10.14426/jsaa.v2i2.80

Rideout, V. J., Foehr, U. G., \& Roberts, D. F. (2010). Generation M2: Media in the lives of 8- to 18-year olds. Henry J. Kaiser Family Foundation. 
Rudasill, K. M., Niehaus, K., Crockett, L. J., \& Rakes, C. R. (2014). Changes in school connectedness and deviant peer affiliation among sixth-grade students from high-poverty neighborhoods. The Journal of Early Adolescence, 34(7), 896-922. https:/doi. org/10.1177/0272431613511330

Silva, T. O., \& Silva, L. T. (2017). Os impactos sociais, cognitivos e afetivos sobre a geração de adolescentes conectados às tecnologias digitais. Revista Psicopedagogia, 34(103), 87-97. http://pepsic. bvsalud.org/pdf/psicoped/v34n103/09.pdf

United Nations Children's Emergency Fund (2016). Early and late adolescence. UNICEF. http://www.unicef.org/ sowc2011/pdfs/Early-and-late-adolescence.pdf

Wendt, G. W. (2012). Cyberbullying em adolescentes brasileiros. Dissertação de Mestrado (Psicologia Clínica), Universidade do Vale do Rio dos Sinos, São Leopoldo.
Wendt, G. W., \& Lisboa, C. (2014). Adolescência nos contextos das tecnologias de informação e comunicação. In L. F. Habigzang, E. Diniz, \& S. H. Koller (Eds.), Trabalhando com Adolescentes: Teoria e Intervenção psicológica (pp. 180-190). ArtMed.

Yen, C. F., Ko, C. H., Yen, J. Y., Chang, Y. P., \& Cheng, C. P. (2009). Multi-dimensional discriminative factors for Internet addiction among adolescents regarding gender and age: Internet addiction in adolescence. Psychiatry and Clinical Neurosciences, 63(3), 357-364. https:/doi.org/10.1111/j.1440-1819.2009.01969.x

Zaremba, R., Romão-Dias, D., \& Nicolaci-da-Costa, A. M. (2002). Simples como uma torradeira: Um estudo sobre o computador no cotidiano da nova geração. Psicologia: Ciência e Profissão, 22(1), 92-99. https:/ doi.org/10.1590/S1414-98932002000100010

Data de submissão: $12 / 02 / 2019$ Primeira decisão editorial: 22/04/2019 Aceite em 14/06/2019 